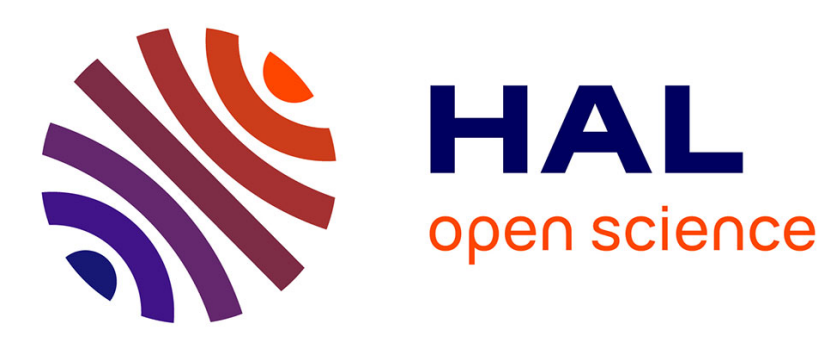

\title{
Comparative Study of Two Homeostatic Mechanisms in Evolved Neural Controllers for Legged Locomotion
}

\author{
Thierry Hoinville, Patrick Henaff
}

\section{To cite this version:}

Thierry Hoinville, Patrick Henaff. Comparative Study of Two Homeostatic Mechanisms in Evolved Neural Controllers for Legged Locomotion. 2004 IEEE/RSJ International Conference on Intelligent Robots and Systems, Oct 2004, Japan. pp.2624-2629. hal-00523324

\section{HAL Id: hal-00523324 \\ https://hal.science/hal-00523324}

Submitted on 4 Oct 2010

HAL is a multi-disciplinary open access archive for the deposit and dissemination of scientific research documents, whether they are published or not. The documents may come from teaching and research institutions in France or abroad, or from public or private research centers.
L'archive ouverte pluridisciplinaire HAL, est destinée au dépôt et à la diffusion de documents scientifiques de niveau recherche, publiés ou non, émanant des établissements d'enseignement et de recherche français ou étrangers, des laboratoires publics ou privés. 


\title{
Comparative Study of Two Homeostatic Mechanisms in Evolved Neural Controllers for Legged Locomotion
}

\author{
Thierry Hoinville and Patrick Hénaff \\ LIRIS \\ Versailles Saint-Quentin-en-Yvelines University, CNRS \\ VÉLIZY, FRANCE \\ Email: thierry.hoinville@liris.uvsq.fr
}

\begin{abstract}
This paper presents a preliminary study on the advantages of two bio-inspired homeostatic mechanisms in neural controllers of legged robots. We consider a robot made up of one leg of 3 dof pushing a body that is sliding on a rail with a friction force. The synthesis of the controller is done by an evolutionary algorithm which choose to attach to each synapse a particular plastic law. Four models of network incorporating or not each homeostatic law are proposed. After evolution, effectiveness of each kind of adaptive controllers is compared in term of statistics on a task of controlling the speed of the robot. The robustness to a perturbation generated by the viscous friction is analyzed in term of control. Results show that homeostatic mechanisms increase evolvability, stability and adaptivity of those controllers.
\end{abstract}

\section{INTRODUCTION}

In the field of legged robots, evolving neural controllers has been successfully applied to various problems [7], [12], [14], [16]. In the majority of the cases, these kind of controllers are able to control the legs of the robot (hexapod or quadripod) to move it following a desired velocity or a target without holding account internal and environmental perturbations like transmission breaking, loss of legs or loss of adherence (see figure 1). Our research

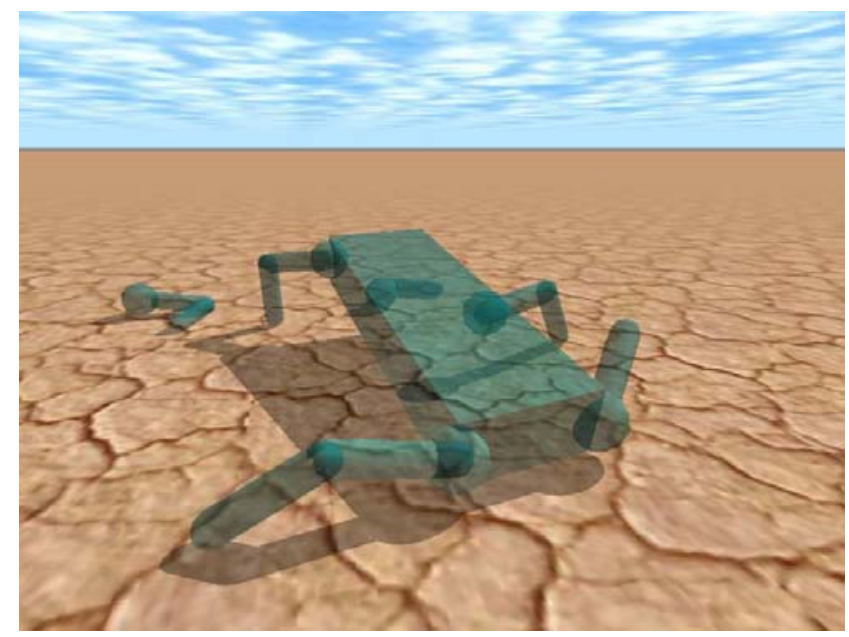

Fig. 1. An hexapode with two lost legs: how could we make a control system adaptive to such a situation? project named IRON ${ }^{1}$, is intended to increase the autonomy and the robustness of robots by confronting evolutionary adjusted neural controllers with different kinds of perturbations. A promising way to do this is to incorporate to neural controllers some plasticity mechanisms which are inspired from biology [6]. Nevertheless, this plasticity might not be sufficient because it tends to destabilize those controllers.

In this paper, we achieve a comparative study of the effects of two bio-inspired homeostatic mechanisms on the evolvability of plastic neural controllers embodied in a single-legged robot. Then, we will analyse the control stability and its adaptivity to an external perturbation.

The paper is organized as follows: the following section presents the robotic problem on which we apply our approach, the third section is devoted to the neuronal models from a biological, mathematical and evolutionary points of view. Then, the next section shows statistical and behavioral results. Thereafter, results and mechanisms modelling are discussed in the next section. Finally, in the last section, we conclude by giving further developments within our project.

\section{ROBOTIC PROBLEM}

The aim of our project is to provide to a multi-legged robot a neuro-controller, synthesized by evolution, that should adapt its behavior to perturbations. These perturbations can be external (environment) or internal (mechanical or electrical faults). However, a main issue of evolutionary robotics, called the scalability problem, is the application of its methods to systems that show a high degree of complexity. Also, to compare the four neural models described in section III-B, we apply our approach to a single-legged robot simulation ${ }^{2}$.

The robot (figure 2) is composed by a body and a leg endowed with three degrees of freedom: two for the hip and one for the knee (see table I for mass and geometrical

\footnotetext{
${ }^{1}$ Implémentation RObotique de Neuro-contrôleurs adaptatifs (http: //www.liris.uvsq.fr/iron/Iron.html). This project, initiated with J.-A. Meyer from the AnimatLab, is supported by the ROBEA program of the CNRS (http://www.laas.fr/robea).

${ }^{2}$ Based on a simulator called Open Dynamic Engine (http: //q12 . org/ode/)
} 

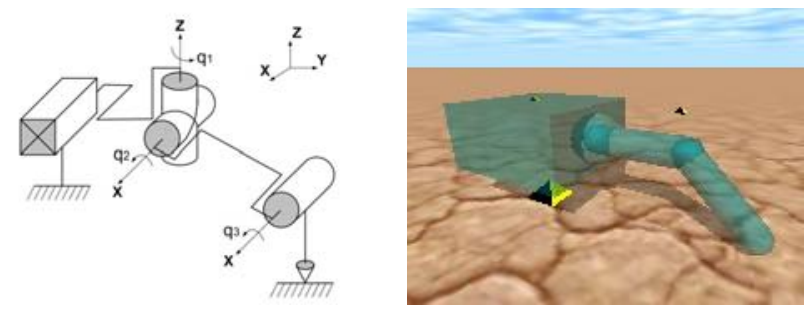

Fig. 2. Morphology of the single-legged robot. Left: Kinematic model. Right: Simulation view.

parameters). A binary contact sensor is fixed on the leg tip and on each joint, a servo-motor ordering its angular position is simulated.

TABLE I

MECHANICAL PARAMETERS OF THE ROBOT

\begin{tabular}{|l|l|l|l|}
\hline Part & Shape & Mass $[\mathrm{kg}]$ & Dimension $[\mathrm{cm}]$ \\
\hline \hline body & box & 3 & $20 \times 20 \times 10$ \\
\hline hip & sphere & 0.5 & $\varnothing 8$ \\
\hline thigh & $\begin{array}{l}\text { capped } \\
\text { cylinder }\end{array}$ & 0.5 & $15 \times \varnothing 4$ \\
\cline { 1 - 3 } shank & \multicolumn{2}{|c|}{} \\
\cline { 1 - 2 }
\end{tabular}

A prismatic link connecting the robot body to the ground, constrains its movements by guiding it on $\vec{X}$ axis. A viscous friction force is applied to the robot. This force $F_{f r}$ is equal to $-k_{f r} V_{e f f}$ where $V_{e f f}$ is the effective speed of the robot body's centre of mass and $k_{f r}$ is the viscous coefficient.

The task of the robot is to respect a desired walk speed $V_{\text {des }}$ and to simultaneously offset a potential perturbation which consists of varying the coefficent $k_{f r}$. This perturbation, external from the leg's point of view, could simulate, from a multi-legged robot's point of view, an internal perturbation as a mass growth or a disruption of another leg.

The performance $p$, of a controller is evaluated at the end of a simulation of $T=10 \mathrm{sec}$. according to the following expression:

$$
p=\frac{1}{T} \int_{0}^{T}\left|V_{d e s}-\widetilde{V_{e f f}}\right| d t
$$

where $\widetilde{V_{e f f}}$ is the global walk speed of the $\operatorname{robot}^{3}$.

\section{METHODS}

\section{A. Homeostatic mechanisms in biological neurons}

Historically, research in neurophysiology are first centered on synaptic plasticity [10]. The mechanisms controlling this plasticity are commonly considered as the main vector of information storage in neural networks and synaptic connection refinement during cerebral development. Thus, by establishing correlation between simultaneous active neurons, Hebbian rules allow neural

\footnotetext{
${ }^{3}$ Temporal average calculed by application of a second order low-pass filter on $V_{e f f}$. This value is preferred to $V_{e f f}$, subject to high amplitude variations at the time of each stride.
}

circuits to adapt to received information. Nevertheless, these flexibility mechanisms are source of instability [15]. Recents studies [18], show that they are associated with homeostatic rules which regulate intrinsic properties of each neuron. In this paper, we are interested in two kinds of them: one regulating neuronal excitability and the other stabilizing total synaptic input strength of each neuron.

1) Regulation of excitability: Excitability of a neuron, i.e. its propensity to transmit more or less action potentials according to information it gets, depends on concentrations of various molecules which are present in its cell body and in its closed vicinity. Ion channels inserted in its membrane actively regulate these concentrations. According to Desai and al. [3], this regulation seems to be driven by the average activity of the cell. Thus, when activity of a neuron is high, its excitability decreases to return to a functional firing rate. Conversely, if the cell tends to be silent, its excitability increases until its firing rate gets back to a functional range (top of figure 3 ).
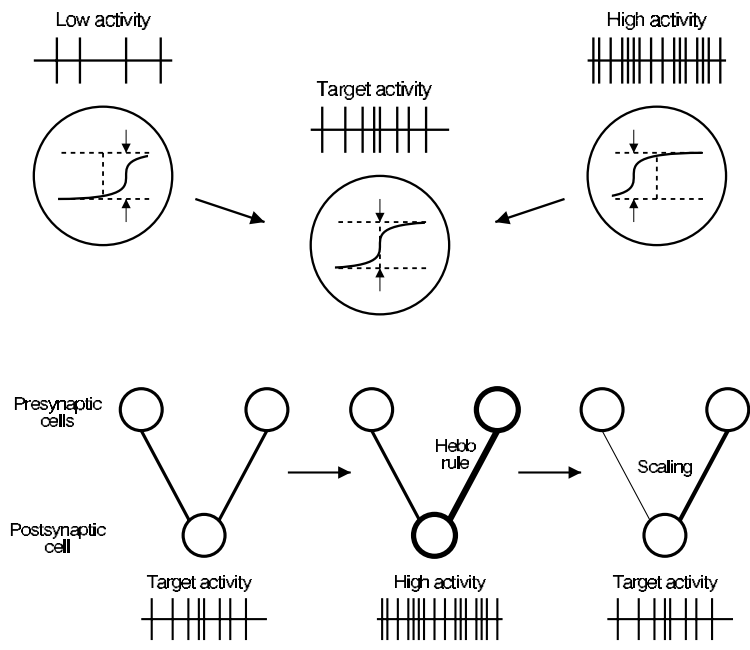

Fig. 3. Two homeostatic mechanisms regulating intrinsic properties of neurons. Circles represents nerve cells. (These figures are directly inspired from the work of Turrigiano [18]) Top: Regulation of excitability The cell's activation threshold is regulated according to its own activity. Bottom: Multiplicative scaling of all synaptic inputs strengths. After an hebbian potentiation of a synapse, the scaling mechanism induces synaptic competition on postsynaptic cell's inputs. Bold circles symbolize activated neurons. Line widths indicate the strength of the corresponding connection.

2) Stabilization of total synaptic strength: During development and learning, synapses number and properties are submitted to deep changing. These modifications can severely alter activity patterns of neurons. According to Turrigiano and al. [19], to preserve their functionalities, neurons regulate their total synaptic input strength. Thus, the excitations amplitude remains in a relevance domain (bottom of figure 3). Experimental observations tend to state that this regulation is applied multiplicatively and globally to all synaptic inputs of a related cell. By its multiplicative nature, this mechanism ensures relative weights of the different connections. Otherwise, far from neutralizing individual synaptic plasticity, this process induces compe- 
tition: if some connections are potentiated, strengths of the others have to decrease.

\section{B. Neuron and synapse model}

In the following, we propose a generic and versatile neuronal model allowing the comparative study of the two homeostatic mechanisms presented above. By versatile, we mean a model that allows to turn each mechanism on or off, independently of the other one. It should be noted that these mechanisms, naturally dynamic and activity-dependent, are formalized here in a static way. Since the time constant of these mechanisms is much higher than those of the activation and learning processes, we assume this is an acceptable simplification.

Nerve cells composing our neural controller are based on a leaky integrator model [2]:

$$
\begin{aligned}
\tau_{i}^{n e u} \frac{d y_{i}}{d t} & =-y_{i}+\sum_{j=1}^{N_{i}} w_{i j} o_{j}-\theta_{i}+I_{i} \\
o_{i} & =\frac{1}{1+e^{-\alpha_{i} y_{i}}}
\end{aligned}
$$

where $y_{i}$ represents the mean membrane potential of the neuron $i$ and $o_{i}$ its activity. $w_{i j}$ is the synaptic strength of the connection from neuron $j$ to neuron $i, N_{i}$ is the number of synaptic inputs of the neuron $i$ and $\tau_{i}^{\text {neu }}$ denotes the time constant of the membrane potential. $I_{i}$ corresponds, in the case of the cell $i$ is a sensory neuron, to an external excitation coming from a sensor. Finally, $\alpha_{i}$ is a gain determining the slope of the sigmoidal activation function and $\theta_{i}$ is the threshold of the neuron's activity.

The formal description of the synapse model results directly from the work of Floreano and al. [5] in which a local adaptation rule is assignated to each connection as suggested by biological observations:

$$
\tau_{i j}^{s y n} \frac{d\left|w_{i j}\right|}{d t}= \begin{cases}\text { Plain Hebb rule } \\ \left(1-\left|w_{i j}\right|\right) o_{j} o_{i} \\ \text { Pre-synaptic rule } \\ \left(1-\left|w_{i j}\right|\right) o_{j} o_{i}+\left|w_{i j}\right| o_{j}\left(o_{i}-1\right) \\ \text { Post-synaptic rule } \\ \left(1-\left|w_{i j}\right|\right) o_{j} o_{i}+\left|w_{i j}\right|\left(o_{j}-1\right) o_{i} \\ \text { Covariance rule } \\ \begin{cases}\left(1-\left|w_{i j}\right|\right) \delta\left(o_{j}, o_{i}\right) & \text { if } \delta\left(o_{j}, o_{i}\right)>0 \\ \left|w_{i j}\right| \delta\left(o_{j}, o_{i}\right) & \text { otherwise }\end{cases} \end{cases}
$$

where $\delta\left(o_{j}, o_{i}\right)=\tanh \left(4\left(1-\left|o_{j}-o_{i}\right|\right)-2\right)$ is a measure of the difference between $o_{j}$ and $o_{i} \cdot \tau_{i j}^{s y n}$ is the time constant of the corresponding learning law (comparable to the learning rate, $\eta$, in the Floreano's model). Note that, in this model, values of synaptic weights are contained in the interval $[-1,1]$.

In order to model the mechanism that regulate neuronal excitability, we use the model of center crossing networks proposed by Mathayomchan and al. [13]. This paradigm consists in determining the ideal activation threshold of a neuron according to its synaptic input weights.

$$
\theta_{i}=\frac{1}{2} \sum_{j=1}^{N_{i}} w_{i j}
$$

Thus, the operating range of each neuron is centered about the most sensitive region of its activation function. Indeed, due to the sigmoid asymmetry (about the $\mathrm{x}$-axis), the excitation range of a neuron can be shifted according to the weight values of its synaptic inputs.

We adapt this concept to build our versatile model by reformulating it. Thus, after transformation ${ }^{4}$ and homogenization $^{5}$, we obtain the modified activity $\widetilde{o}_{i}$ as follows:

$$
\widetilde{o}_{i}= \begin{cases}2\left(o_{i}-\frac{1}{2}\right) & \text { Center-crossing model } \\ o_{i} & \text { Classical model }\end{cases}
$$

Moreover, in order to generalize the model by enabling several types of neurons (ones sensitive to weak excitations, others sensitive to strong excitations), we introduce a new activation threshold, $\theta_{i}^{*}(7)$, independent of the synaptic input strengths of the related neuron.

On the other hand, the mechanism that regulates total synaptic input strengths is modeled by a multiplicative normalization of $\left\|\vec{w}_{i}\right\|=\sqrt{\sum_{j=1}^{N_{i}} w_{i j}^{2}}$ [8]. The synaptic weights are updated according to the following expression:

$$
\left|w_{i j}^{t+d t}\right|= \begin{cases}\frac{\left|w_{i j}^{t}\right|+d\left|w_{i j}^{t}\right|}{\left\|\vec{w}_{i}^{t}+d \vec{w}_{i}^{t}\right\|} & \text { Normalized synapses } \\ \left|w_{i j}^{t}\right|+d\left|w_{i j}^{t}\right| & \text { Classical synapses }\end{cases}
$$

Finally, to abstract the model from the value of $N_{i}$, we define a coefficient, $\mu_{i}$, normalizing the sum $\sum_{j=1}^{N_{i}} w_{i j} \widetilde{o}_{j}$, as follows:

$$
\mu_{i}= \begin{cases}\sqrt{N_{i}} & \text { Normalized synapses } \\ N_{i} & \text { Classical synapses }\end{cases}
$$

Using the above definitions, the general expression of our neuronal model is:

$$
\begin{aligned}
\tau_{i}^{n e u} \frac{d y_{i}}{d t} & =-y_{i}+\frac{1}{\mu_{i}} \sum_{j=1}^{N_{i}} w_{i j} \widetilde{o}_{j}-\theta_{i}^{*}+I_{i} \\
o_{i} & =\frac{1}{1+e^{-\alpha_{i} y_{i}}}
\end{aligned}
$$

This versatile model allows instantiation of four submodels:

- center-crossing model with normalized synapses (CCNS),

- center-crossing model (CC),

- normalized synapses model (NS),

- classical model (CM).

$$
\begin{aligned}
& { }^{4} \sum w_{i j} o_{j}-\theta_{i}=\sum w_{i j} o_{j}-\frac{1}{2} \sum w_{i j}=\sum w_{i j}\left(o_{j}-\frac{1}{2}\right) \\
& { }^{5}\left(o_{j}-\frac{1}{2}\right) \text { is multiplied by } 2 \text { to preserve the same range of values } \\
& \left(\left|\widetilde{o}_{j}\right| \in[0,1]\right) \text { in any model used. }
\end{aligned}
$$




\section{Neural controller structure}

Figure 4 represents an example of an evolved controller. We arbitrarily fix the network size to eight neurons. Two neurons receive sensorial information from the environment, the first one is excited with the consign error $V_{d e s}-$ $V_{e f f}$, the second one is connected to the contact sensor. Three motoneurons drive angular positions of servomotors. The three remaining neurons form the hidden layer of the network.

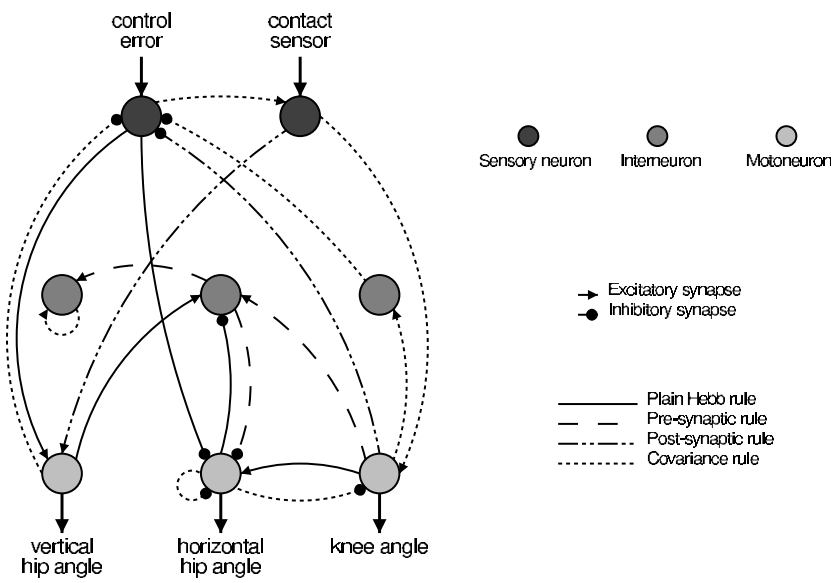

Fig. 4. Example of the phenotype structure of an evolved controller.

Controllers are genetically encoded with numerical and symbolic alleles strings divided in eight neuronal blocks. Figure 5 shows structure of the neuron genotype. A neu-

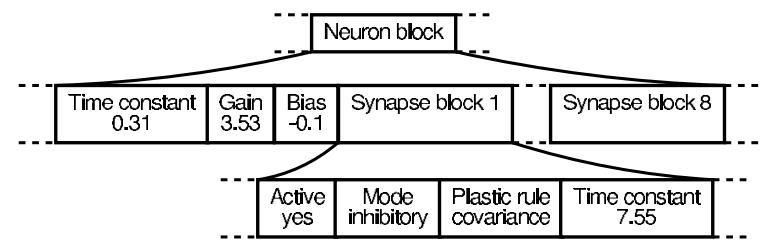

Fig. 5. Structure of the neuron genotype.

ronal block is composed of a list of intrinsic neuronal parameters $\left(\tau^{n e u}, \alpha\right.$ et $\left.\theta^{*}\right)$ and a list of eight synaptic blocks defining properties of cell's output connections with others neurons (including itself). The first synaptic block gene condition the network structure by activating or not the related connection. The next gene indicates its excitatory or inhibitory mode. The two remaining genes dictate the connection dynamic by associating to it one of the four learning rules showed above and the related time constant, $\tau^{s y n}$. The value of each numerical gene is taken from a five length allele set. Table II shows these allele sets.

From a complexity point of view, each genotype is provided with $8 \times(3+8 \times 4)=280$ genes and the size of the genotype search space is $\left(5 \times 5 \times 5 \times(2 \times 2 \times 4 \times 5)^{8}\right)^{8}=3.74 \times 10^{138}$ possibilities.
TABLE II

ALLELES SETS OF NUMERICAL GENES

\begin{tabular}{|l|lllll|}
\hline Gene & \multicolumn{5}{|c|}{ Alleles set } \\
\hline \hline$\tau^{\text {neu }}$ & 0.02, & 0.165, & 0.31, & 0.455, & 0.6 \\
\hline$\alpha$ & 2.46, & 3.53, & 5.34, & 9.43, & 31.26 \\
\hline$\theta^{*}$ & -0.2, & -0.1, & 0, & 0.1, & 0.2 \\
\hline$\tau^{\text {syn }}$ & 0.2, & 2.65, & 5.1, & 7.55, & 10 \\
\hline
\end{tabular}

\section{Evolutionary approach}

Controllers are evolved by a generational and elitist genetic algorithm. Genetic operators are the allelic mutation $\left(P_{\text {mut }}=0.001\right)$ and the uniform crossover $\left(P_{\text {cross }}=\right.$ $0.6)$. Individuals are selected by the stochastic universal sampling algorithm [1] according linearly to their rank in the population [9] (with the best individual producing an average 1.1 offspring).

During evolution, each controller is evaluated through three successive simulations with different scenarios. As we can see on table III, a scenario is defined by temporal variations of $V_{d e s}$ and $k_{f r}$ parameters. The scenario A

TABLE III

THREE EVALUATION SCENARIOS

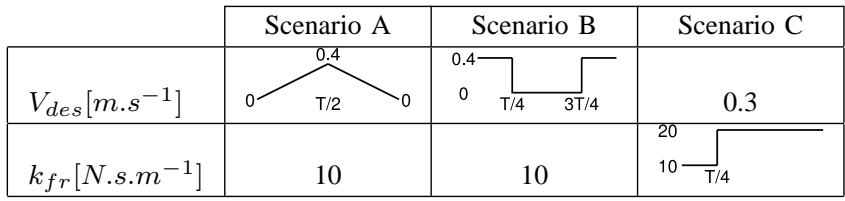

corresponds to a simple control behavior, the scenario B reward the capacity of inhibiting robot gait and the scenario $\mathrm{C}$ favours adaptation to the perturbation. The global fitness of an individual is the quadratic combination of the three elementary scores obtained from these evaluations (the lower the fitness is, the better the controller behaves).

$$
\text { fitness }=\sqrt{p_{A}^{2}+p_{B}^{2}+p_{C}^{2}}
$$

This kind of combination restricts the compensation effect produced by a classical average and supports behaviors that include the three qualities described by the scenarios.

\section{RESUlts}

\section{A. Statistical analysis}

For each neuronal models, we performed 10 evolution runs with different random initializations of populations of 200 individuals. Populations are evolved during 2000 generations. Figure 6 shows statistical results of this experiment in term of best fitness. From these data, we draw the following main results:

- Compared with the classical model (CM), both homeostatic mechanisms (CC and NS) clearly improve evolvability of the controllers, either in final solution or in evolution speed.

- CC model is, on average, more efficient than NS model, but results variability indicates that evolution 


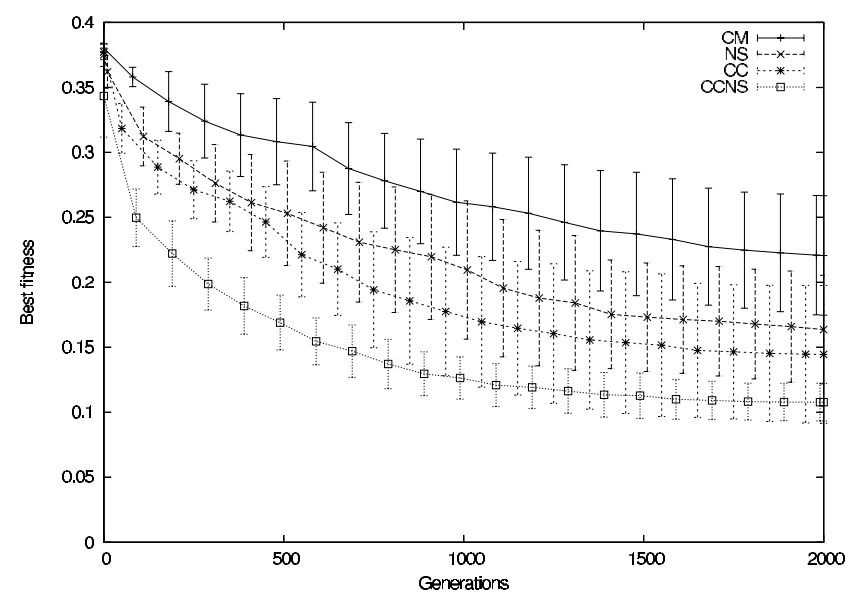

Fig. 6. Best fitness for each neuronal model averaged over 10 runs with different random initializations of the population.

of CC controllers strongly depends on initial random populations.

- CCNS model is the most performant model and always results in high fitness controllers (low value).

To verify relevance of the homeostatic mechanisms to stabilize neural networks, we avaluated the long-term behavior of the 10 best controllers for each neuronal model. To do this we applied to those controllers a constant desireed speed of $0.3 \mathrm{~m} \cdot \mathrm{s}^{-1}$. The stability test is succeeded if the robot reach the distance of $150 \mathrm{~m}$ in less than $1000 \mathrm{~s}$. In case of perfect controllers this distance must be reach in $500 s$. Controllers are tested in non-perturbed $\left(k_{f r}=10 N . s . m^{-1}\right)$ and perturbed $\left(k_{f r}=20 N . s . m^{-1}\right)$ environments (the perturbation occurs at $t=250 \mathrm{~s}$ ). Success rates and average times spent to reach the given distance in case of success are indexed in table IV. These

TABLE IV

STABILITY OF EACH CONTROLLER MODEL

\begin{tabular}{|l|c|c|c|c|}
\hline \multirow{2}{*}{ Model } & \multicolumn{2}{|c|}{ Non-perturbed } & \multicolumn{2}{c|}{ Perturbed } \\
\cline { 2 - 5 } & Success[\%] & Avg. time[s] & Success[\%] & Avg. time[s] \\
\hline \hline CCNS & 90 & 537 & 80 & 586 \\
\hline CC & 64 & 576 & 37 & 675 \\
\hline NS & 72 & 597 & 70 & 669 \\
\hline CM & 55 & 792 & 20 & 849 \\
\hline
\end{tabular}

results corroborate the previous fitness observations except for relative performances of CC and NS models. Indeed, prolonged-time simulations appear to favour models which incorporate normalized synapses, especially in perturbed environment.

\section{B. Control analysis}

To complete the above statistical results, we achieved behavioral and functionnal analysis of the best controllers. During these simulations, a periodic squared signal is submitted to the robot as desired speed while a perturbation occurs. Figure 7 shows an example of time-plots of the desired and the effective speed obtained for the best CCNS controller (figure 4). Friction coefficient and joint commands computed by the neural network are also plotted. From these plots, we can see that control task is satisfied

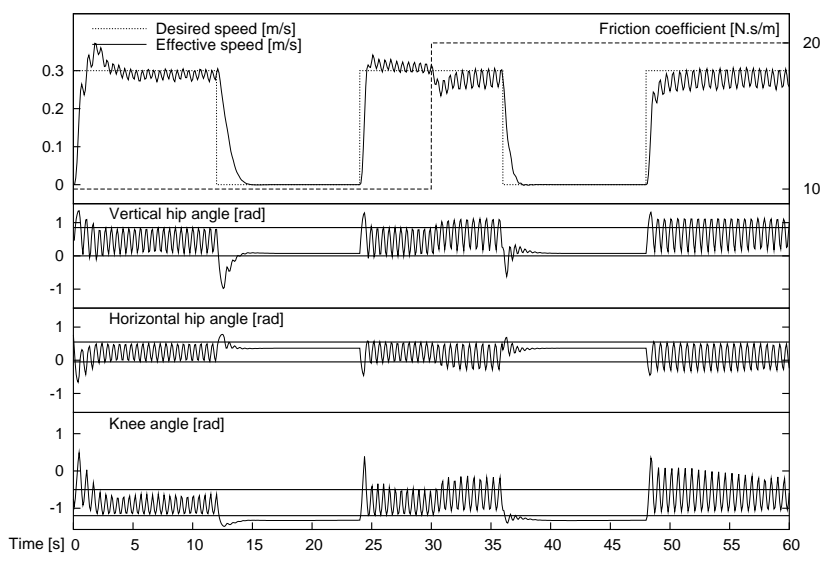

Fig. 7. Time-plots of $V_{d e s}, \widetilde{V_{e f f}}, k_{f r}$ and joint commands for a CCNS controller. The robot is perturbated at $t=30 \mathrm{sec}$. Oscillations of the effective speed correspond to the robot strides.

even when the perturbation occurs. The perturbation does not significantly alter the robot speed and the control task error remaining relatively small. Moreover, the adaptation to the perturbation is confirmed by time-plots of joint commands. Increasing of the friction force causes the amplification of signals send to servo-motors (particularly obvious on the knee joint). Note that walking frequency is not altered by the perturbation. In addition, we can see

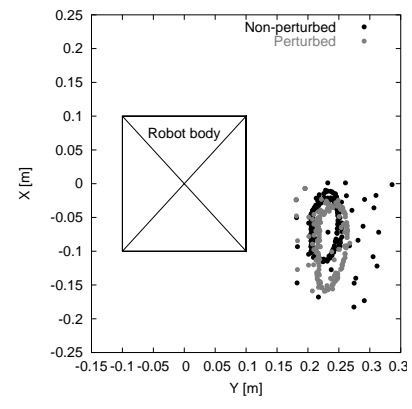

(a) Horizontal projection

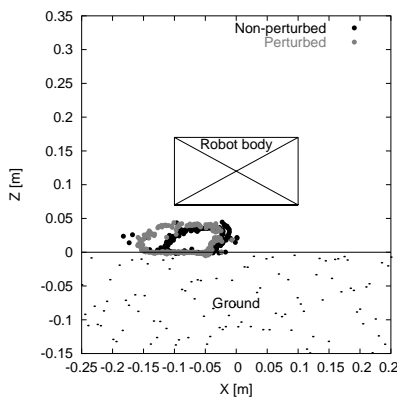

(b) Vertical projection
Fig. 8. Cartesian trajectories of the end of the robot leg with or without perturbation

on figure 8 that the robot gait is adapted in perturbed environment. Indeed, the robot strides are lengthened and shifted back from the body, this leg configuration increases the resulting force when contact with the ground occurs. Finally, we show, on the figure 9, the time-plots of synaptic strengths of the corresponding neural network. This figure indicates that the network adjusts its synaptic strengths according to the desired speed and the perturbation.

\section{Discussion}

As the above results show, when simulation time is prolonged, NS controllers are more efficient than CC 


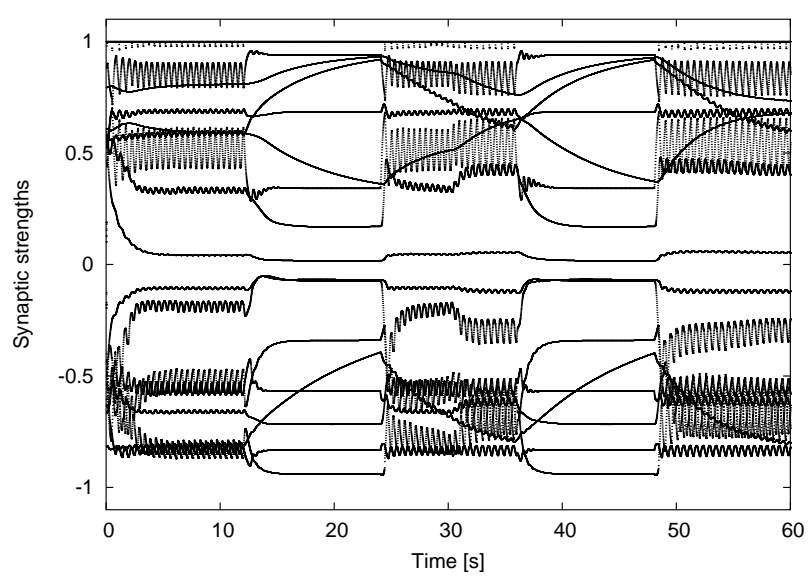

Fig. 9. Time-plots of synaptic strengths

controllers. By analysing long-term behavior of the both model, it can be seen that normalized synapses contribute to stabilize neural networks function. Indeed, CC and CM controllers produce less robust oscillatory patterns. Preliminary obervations indicate that these neural networks cannot reconfigure their synaptic strengths after an inhibition or a perturbation of the robot gait. We suppose that the synaptic normalization constraint favours dynamical stability of neuronal systems. Also, this could explain the low variance of NS and CCNS evolution results (figure 6).

Either during evolution (figure 6) or at post-evolutionary evaluations (table IV), statistical results show that CCNS model is clearly more efficient than CC and NS models. Since their association is more powerful than their respective individuality, we can state that there is a synergy between the both mechanism.

In our model, the synaptic normalization is expressed in a static way. This assumption is made due to the fact that, from a biological point of view, this mechanism is relatively slow. However, it should be interesting to study its dynamic modelling as in the Oja's learning rule [17]. Moreover, by its local nature, this rule is more biologically plausible. Likewise, a dynamical regulation of neurons excitability could be more powerful by allowing a more wider range of dynamics.

\section{CONCLUSION AND FURTHER WORK}

In this paper, we show that evolvabilty, in term of both speed and final result, of plastic neural controllers is improved by including to them bio-inspired homeostatic mechanisms that regulate neuronal excitability. Evolved controllers show increased stability and perturbation adaptivity. On the other hand, since our neuronal model is not specifical to the task we test it on, our results suggest that these mechanisms could be benefical to others applications or approach (back-propagation, reinforcement learning, etc).

Within the scope of the IRON project, the aim of our current work is to extend our approach to robots of several morphologies undergoing various kind of perturbations. To this end, the addition of a dynamic system, driving intrinsic neuronal properties and synaptic plasticity, could improves neural controllers adaptivity by allowing them to reconfigurate themself. A potential issue is to integrate to our neural model some bio-inspired paradigms based on chemical messengers [4], [11].

\section{REFERENCES}

[1] J. E. Baker. Reducing bias and ineffiency in the selection algorithm. In Lawrence Erlbaum Associates, editor, Proc. of the second Int. Conf. on Genetic Algorithms and their Applications, Hillsdale, 1987.

[2] R.D. Beer. On the dynamics of small continuous-time recurrent neural networks. Adaptive Behavior, 3(4):469-509, 1995.

[3] N.S. Desai, L.C. Rutherford, and G.G. Turrigiano. Plasticity in the intrinsic excitability of neocortical pyramidal neurons. Nature Neuroscience, 2(6):515-520, 1999.

[4] P. Eggenberger, A. Ishiguro, S. Tokura, T. Kondo, and Y. Uchikawa. Toward seamless transfer from simulated to real worlds: A dynamically-rearranging neural network approach. In J. Wyatt and J. Demiris, editors, Proc. of the Eighth Eur. Workshop on Learning Robots, pages 4-13, EPFL, Lausanne, Switzerland, 1999.

[5] D. Floreano and J. Urzelai. Evolution of neural controllers with adaptive synapses and compact genetic encoding. In 5th European Conf. on Artificial Life, 1999.

[6] D. Floreano and J. Urzelai. Evolutionary robotics: The next generation. In T. Gomi, editor, Evolutionary Robotics III, Ontario (Canada), 2000. AAI Books.

[7] J.C. Gallagher, R.D Beer, K.S. Espenschied, and R.D. Quinn Application of evolved locomotion controllers to a hexapod robot. Robotics and Autonomous Systems, 19:95-103, 1996.

[8] W. Gerstner and W.M. Kistler. Spiking Neuron Models, chapter 11. Cambridge Univ. Press, 2002. (http://diwww.epfl.ch/ gerstner/BUCH.html).

[9] D. E. Goldberg. Genetic algorithms in search, optimization, and machine learning. Addison-Wesley, Reading, MA, 1989.

[10] D.O. Hebb. The Organization of Behavior: A Neurophysiological Theory. John Wiley and Sons, 1949.

[11] P. Husbands, T.M.C. Smith, N. Jakobi, and M. O'Shea. Better living through chemistry: Evolving gasnets for robot control. Connection Science, 10(3-4):185-210, 1998

[12] A. J. Ijspeert, J. C. T. Hallam, and D. J. Willshaw. From lampreys to salamanders: Evolving neural controllers for swimming and walking. In From Animals to Animats: Proc. of the Fifth Int. Conf. of the The SAB. MIT Press, 1998.

[13] B. Mathayomchan and R.D. Beer. Center-crossing recurrent neural networks for the evolution of rhythmic behavior. Neural Computation, 14:2043-2051, 2002.

[14] J.-A. Meyer, S. Doncieux, D. Filliat, and A. Guillot. Biologically inspired robot behavior engineering. In R.J. Duro, J. Santos, and M. Graña, editors, Evolutionary Approaches to Neural Control of Rolling, Walking, Swimming and Flying Animats or Robots. Springer-Verlag, 2002.

[15] K.D. Miller and D.J.C. MacKay. The role of constraints in hebbian learning. Neural Computation, 6:100-126, 1994.

[16] S. Nolfi. Evolving non-trivial behaviors on real robots: a garbage collecting robot. Robotics and Autonomous System, 22:187-198, 1997.

[17] E. Oja. A simplified neuron model as a principal component analyzer. Math. Biol., 15:267-273, 1982.

[18] G.G. Turrigiano. Homeostatic plasticity in neuronal networks: the more things change, the more they stay the same. Trends in Neuroscience, 22(5):221-228, 1999.

[19] G.G. Turrigiano, K.R Leslie, N.S Desai, L.C. Rutherford, and S.B. Nelson. Activity-dependent scaling of quantal amplitude in neocortical pyamidal neurons. Nature, 391:892-895, 1998. 